\title{
Pharmacological Treatment for Neonatal Abstinence Syndrome is Associated with Altered DNA Methylation and Neurobehavior
}

Marie Camerota $\mathrm{PhD}^{1,2}$, Jonathan M. Davis $\mathrm{MD}^{3}$, Lynne M. Dansereau MSPH${ }^{2}$, Erica L.

Oliveira BA ${ }^{2}$, James F. Padbury MD ${ }^{2}$, Barry M. Lester $\mathrm{PhD}^{1,2}$

${ }^{1}$ Department of Psychiatry and Human Behavior, Alpert Medical School of Brown University, Providence, RI, USA

${ }^{2}$ Department of Pediatrics, Women and Infants Hospital, Providence, RI, USA

${ }^{3}$ Department of Pediatrics, Tufts Children's Hospital and the Clinical and Translational Science Institute, Boston, MA, USA

Corresponding Author: Correspondence concerning this article and requests for reprints should be addressed to Marie Camerota, Women and Infants Hospital of Rhode Island, 101 Dudley Street, Providence, RI 02905; email: marie_camerota@brown.edu; telephone: 401-274-1122 ext. 48905 .

Financial Support: This work was funded by the National Institutes of Drug Abuse (NIDA) grant R21DA041706-02 (JMD, BML) and R01DA032889 (JMD, BML) and by the Charles H. Hood Foundation (JMD, BML). MC was additionally supported by an institutional training grant from the National Institutes of Mental Health (NIMH), grant T32MH019927. Study funders had no role in study design, data collection, data analysis, interpretation of study results, or writing of the manuscript.

Disclosures: The authors declare no conflicts of interest.

Keywords: NAS; DNA methylation; OPRM1; opioids; neurobehavior, NICU Network Neurobehavioral Scales

\section{List of Abbreviations:}

CpG: cytosine-phosphate-guanine

DNAm: DNA methylation

NAS: Neonatal abstinence syndrome

NNNS: NICU Network Neurobehavioral Scales 


\begin{abstract}
Objective: To determine whether pharmacological treatment for neonatal abstinence syndrome (NAS) is associated with changes in DNA methylation (DNAm) of the mu-opioid receptor gene $(O P R M 1)$ and improvements in neonatal neurobehavior.

Study Design: Buccal swabs were collected from 37 neonates before and after morphine treatment for NAS. Genomic DNA was extracted and DNAm was examined at four CpG sites within the OPRM1 gene. The NICU Network Neurobehavioral Scales (NNNS) was also performed before and after NAS treatment. Changes in DNAm (DNAm post-tx - DNAm $\left._{\text {pre-tx }}\right)$ and NNNS summary scores $\left(\mathrm{NNNS}_{\text {post-tx }}-\mathrm{NNNS}_{\text {pre-tx }}\right)$ were then calculated. Path analysis was used to examine associations among pharmacologic treatment (length of treatment and total dose of morphine), changes in DNAm, and changes in NNNS summary scores.
\end{abstract}

Results: DNAm significantly decreased from pre- to post-treatment at 1 of $4 \mathrm{CpG}$ sites within the $O P R M 1$ gene. Neonates also demonstrated decreased excitability, hypertonia, lethargy, signs of stress and abstinence, and increased quality of movement and regulation from pre- to posttreatment. Increased length of treatment and higher morphine doses were associated with greater decreases in DNAm; greater decreases in DNAm were associated with greater decreases in excitability and hypertonia on the NNNS.

Conclusions: Pharmacological treatment of NAS is associated with decreased DNAm of the OPRM1 gene and improved neonatal neurobehavior. Epigenetic changes may play a role in these changes in neonatal neurobehavior. 


\section{Introduction}

The prevalence of prenatal opioid exposure increased by 333\% from 1999 to 2014 in the United States [1]. This has been accompanied by a steep rise in the incidence of neonatal abstinence syndrome (NAS), from 1.2 to 8.8 per 1,000 hospital births between 2000 and 2016 [2-6]. NAS describes a constellation of signs in the neonate including irritability, inconsolable, high-pitched crying, hypertonia, feeding difficulty, tremors, and gastrointestinal dysfunction (e.g., vomiting, loose stools) [2].

The most common pharmacological treatment for NAS is morphine, with a minority of neonates treated with other opioid replacements [7]. Pharmacological treatment for NAS is associated with improved neurobehavior as assessed by the NICU Network Neurobehavioral Scales (NNNS)[8,9]. Specifically, neonates show improvements in orientation, quality of movement, excitability, hypertonia, and signs of stress (e.g., high pitched cry) over the course of pharmacological treatment. NNNS summary scores have also been linked to maternal buprenorphine dose and have been found to differentiate between neonates who require pharmacological treatment for NAS and those that do not $[10,11]$.

The average length of stay (LOS) for NAS is approximately 12 days [7]. However, there is significant variability in the severity of NAS, including the amount (e.g., total morphine dose) and type(s) of pharmacologic treatment (e.g., adjunctive medication) needed. Genetic and epigenetic factors likely account for some of this variability [12]. Specifically, epigenetic variation in the mu opioid receptor $(O P R M 1)$ gene has been linked to NAS severity [13,14]. It is unknown whether pharmacological treatment for NAS is associated with changes in DNA methylation (DNAm) of OPRM1 and whether these changes are associated with improvements in neurobehavior commonly observed following pharmacologic treatment for NAS. Given that 
increased methylation of $O P R M 1$ is associated with more severe NAS, we hypothesized that pharmacologic treatment for NAS would result in decreased DNAm and improvements in neonatal neurobehavior.

\section{Methods}

\section{Participants}

This study included 37 mothers treated with methadone (43\%) or buprenorphine $(57 \%)$ for an opioid use disorder. Neonates were treated with morphine for NAS at Women and Infants Hospital (Providence, RI). Neonates who exceeded a predetermined opioid dose also received phenobarbital. Exclusion criteria included gestational age $<37$ weeks and evidence of sepsis, major congenital anomalies, or genetic disorders. The mean gestational age of the infants was $38.9 \pm 1.1$ weeks and mean birth weight was $3011 \pm 382 \mathrm{~g}$. The study was approved by the Institutional Review Board at each site and study investigators obtained informed consent.

\section{Measures}

Neonatal Neurobehavior was assessed shortly after birth and at hospital discharge by trained examiners using the NNNS, a standardized neurobehavioral exam that includes neurological integrity, motor and behavioral function, and signs of stress and abstinence [15]. It has been used previously in studies of prenatal drug exposure [8,16-19]. The NNNS results in 12 summary scores (e.g., quality of movement, hypertonia, excitability) including stress/abstinence, and has been used previously in studies of prenatal drug exposure [8,16-19].

DNA was sampled from buccal swabs collected immediately following the NNNS exam shortly after birth and at hospital discharge. Quantitative DNA methylation was measured as previously described [20]. Briefly, genomic DNA was extracted from pooled saliva samples using QIAmp DNA Mini Kits (Qiagen, Inc.) following manufacturer's protocols and purified 
DNA was used for quantitative methylation analysis by pyrosequencing. DNA samples $(1 \mu \mathrm{g})$ were bisulfite-modified using the EZ DNA methylation Kit (Zymo Research, Irvine, CA) following the manufacturer's protocol. Pyrosequencing was performed on a Qiagen Q48 pyrosequencing plantform on PCR product amplified from bisulfite-modified DNA using assay reagents from EpigneDx (Hopkinton, $\mathrm{MA}$ ) for $4 \mathrm{CpG}$ sites in the $O P R M 1$ promoter at positions $(-93,-90,-80,-71)$. The sequence interrogated was CGGCGAAAGGAAGCGGCTGAGGCG.

Dilution series of fully methylated referent DNA into fully unmethylated referent DNA were run with each assay to assess the performance characteristics of the assay.

\section{Statistical Analyses}

Data analysis proceeded in three steps. First, paired samples t-tests determined whether there was significant change in DNAm at the $4 \mathrm{CpG}$ sites within OPRMI before and after pharmacologic treatment. Next, paired samples t-tests were used to test whether there was significant improvement in NNNS summary scores before and after pharmacologic treatment. Finally, path analysis was used to test the full mediated pathway from NAS treatment (i.e., length of treatment [LOT], and total dose of morphine) to change in DNAm of OPRM1, to change in neurobehavior. All path models were run using full-information maximum likelihood to account for missingness in any outcome variables. We adjusted t-tests for multiple testing by using the Benjamini-Hochberg procedure. Results remained significant after imposing a false discovery rate (FDR) of $10 \%$.

\section{Results}

Descriptive statistics for mothers and neonates are shown in Table 1. The sample was split in terms of prenatal exposure to methadone (43\%) or buprenorphine (57\%). A majority of neonates $(75 \%)$ had concurrent exposure to maternal tobacco use. Information about NAS 
treatment is shown in Table 2. Neonates were treated for an average of 14.2 days $(95 \% \mathrm{CI}=12.7$ - 15.8 days) and the average total dose of morphine administered was $13.2 \mathrm{mg}(95 \% \mathrm{CI}=10.5-$ $15.9 \mathrm{mg})$. About half (56\%) of neonates were also treated with phenobarbital.

\section{DNAm before and after NAS treatment}

DNAm significantly decreased from pre- $(M=1.37 \%)$ to post-treatment $(M=0.92 \%)$ at one $\mathrm{CpG}$ site (-71), $t(36)=-2.09, p=.04$ (Table 3).

\section{Neurobehavior before and after NAS treatment}

Neonates exhibited significant decreases in excitability, hypertonia, lethargy, and signs of stress/abstinence and significant increases in quality of movement and regulation from pre- to post-treatment (all $p<.05$; Table 3$)$.

\section{Relationship between changes in DNAm and neurobehavior}

We investigated whether changes in DNAm at the $-71 \mathrm{CpG}$ site were related to changes in NNNS summary scores. Neonates with larger decreases in DNAm at the -71 CpG site had larger decreases in excitability $(r=.40, p=.02)$ and hypertonia $(r=.36, p=.05)$ and greater increases in regulation $(r=-.40, p=.05)$.

\section{NAS Treatment, DNAm, and NNNS outcomes}

A series of path models were tested linking NAS treatment to changes in NNNS scores $(\triangle \mathrm{NNNS})$ via changes in DNAm $(\triangle \mathrm{DNAm})$. Because there was significant change in 6 NNNS subscales (excitability, hypertonia, lethargy, quality of movement, regulation, and stress/abstinence), only these measures were examined. Similarly, we only included DNAm at 1 CpG site (-71) that significantly changed pre- to post-treatment.

Greater LOT $(\beta=-.29, p=.04)$ and total morphine dose $(\beta=-.31, p=.04)$ were associated with greater decreases in DNAm at the $-71 \mathrm{CpG}$ site in all models (Table 4). In the 
LOT model for hypertonia, there was a significant association between LOT and $\triangle \mathrm{DNAm}(\beta=-$ $.29, p=.04)$ and between $\triangle \mathrm{DNAm}$ and $\Delta \mathrm{NNNS}(\beta=.37, p=.001)$. These results indicate that greater LOT was associated with greater decreases in DNAm at the - $71 \mathrm{CpG}$ site and greater decreases in DNAm were in turn associated with greater decreases in hypertonia. The indirect path from LOT to $\triangle \mathrm{DNAm}$ to $\triangle \mathrm{NNNS}$ was not significant $(\beta=-.11, p=.09)$.

A similar pattern emerged in the morphine models. For excitability, greater morphine dose was associated with greater decreases in DNAm $(\beta=-.31, p=.04)$ and greater decreases in DNAm were associated with greater decreases in excitability $(\beta=.40, p=.005)$. The indirect path from morphine dose to $\Delta$ DNAm to $\Delta$ NNNS was significant $(\beta=-.13, p=.04)$. For hypertonia, results were similar to the LOT model. Greater morphine doses were associated with greater decreases in DNAm $(\beta=-.31, p=.04)$. Greater decreases in DNAm were associated with greater decreases in hypertonia $(\beta=.45, p<.001)$. The indirect path from morphine dose to $\triangle$ DNAm to $\triangle$ NNNS was significant $(\beta=-.14, p=.05)$.

\section{Discussion}

The overall goal of this study was to examine whether pharmacologic treatment for NAS was associated with changes in DNAm of OPRM1 and whether changes in DNAm were associated with improvements in neonatal neurobehavior. DNAm significantly decreased from pre- to post-treatment in one $\mathrm{CpG}$ site within OPRMI. Neonates also demonstrated significant improvements in multiple aspects of neurobehavior (e.g., hypertonia, regulation, signs of stress/abstinence) following pharmacologic treatment for NAS. Path models provide support for DNAm as a pathway that may be associated with treatment-related improvements in neonatal neurobehavior. Our findings are novel in that they provide evidence for changes in DNAm 
following pharmacologic treatment for NAS and highlights the potential mechanistic role of DNAm in treatment-related improvements in neurobehavior.

NAS is a highly variable condition whose severity is influenced by a host of factors, including type of antenatal maternal opioid use as well as neonatal genetic and epigenetic factors $[12,19]$. DNAm of the OPRMI gene has been shown to be higher in opioid-exposed versus opioid-naïve neonates [21]. Other studies have demonstrated that greater DNAm of OPRM1 in both mothers and neonates is associated with greater need for pharmacologic treatment for NAS and with a more severe phenotype [13,14]. These studies also demonstrated that hypermethylation (e.g., gene silencing) was associated with the most severe NAS phenotypes. These findings suggests that DNAm of opioid receptor genes is potentially altered by exposure to opioids in utero, with increased DNAm playing a role in the severity of NAS.

Our findings support the hypothesis that pharmacologic treatment for NAS may be beneficial by decreasing DNAm of $O P R M 1$, albeit only at one $\mathrm{CpG}$ site. The CpG site identified in this study (-71) is different from the $\mathrm{CpG}$ sites that have been identified in prior studies as being related to NAS severity (e.g., $-18,-14,-10,+23,+84)[13,14]$ Most of these previouslyidentified CpGs are located within putative transcription factor binding sites, meaning that increased DNAm of these sites may be associated with decreased gene expression [22,23]. Increased DNAm of the OPRMI gene has been shown to decrease protein expression of muopioid receptors which could increase the need for pharmacologic treatment of NAS symptoms $[13,24]$. Despite being located outside of a transcription factor binding site, the $-71 \mathrm{CpG}$ site is the most proximal of all investigated $\mathrm{CpG}$ sites to the $O P R M 1$ promoter region. This suggests that differences in DNAm at this site may have increased biological relevance compared to other sites we investigated. 
Since prenatal opioid exposure is associated with greater DNAm of OPRM1, the decrease in DNAm of the -71 CpG site suggests that pharmacologic treatment may 'correct/reverse' altered patterns of DNAm [21]. Just as greater DNAm is associated with increased NAS severity via reduced gene expression, decreases in DNAm following pharmacologic treatment may be associated with increased gene expression and the amelioration of NAS. The exact mechanism by which changes in DNAm of OPRM1 impact gene expression and the severity of NAS remains to be determined, but these findings provide additional support that epigenetic mechanisms are involved (directly or indirectly) in explaining the efficacy of pharmacologic treatment. The extent to which treatment-related changes in DNAm persist and whether they differ as a function of the specific drug treatment (e.g., morphine, buprenorphine) requires further study.

Neonatal neurobehavior has been extensively studied in relation to prenatal opioid exposure, with studies documenting differences in NNNS summary scores based on opioid exposure and type and dose of maternal opioid (e.g., methadone versus buprenorphine) $[10,11,16,17]$. More recently, NNNS scores have been shown to differentiate neonates with highand low-severity of NAS [25]. Neonates with severe NAS were more likely to have a neurobehavioral profile characterized by poor regulation, high arousal, hypertonicity, and physiological signs of stress and abstinence. Pharmacologic treatment for NAS has been associated with improvements in neonatal neurobehavior [8,9] and lower rates of infant mortality [26]. Indeed, the current study confirmed that there were improvements in neonatal neurobehavior following pharmacologic treatment for NAS. Given that atypical NNNS profiles have been linked to abnormal long-term developmental outcomes, our findings suggest that neonates who show positive changes in NNNS post-treatment may also have better long-term outcomes $[25,27]$. 
Path analysis models suggested that changes in DNAm may underlie the association between pharmacologic treatment for NAS and improvements in neonatal neurobehavior. Neonates who exhibited greater treatment related decreases in DNAm also showed the most significant improvements in excitability and hypertonia. These findings suggest that pharmacologic treatment for NAS is associated with changes in both DNAm and neurobehavior which provides preliminary support for epigenetics as a putative mechanism underlying the efficacy of pharmacologic treatment for NAS. Additionally, a growing number of studies have shown that DNAm may be one predictor of individual differences in neonatal neurodevelopment [28,29]. By linking changes in DNAm to neonatal neurobehavior, this study provides stronger support for the putative role of DNAm as a determinant of neurobehavior.

Several limitations of this study should be noted. First, our small sample size may have limited our power to detect small changes in DNAm and neurobehavior, or to detect relatively small associations between DNAm and neurobehavior. However, the significant associations reported are of medium to large effect sizes meaning that they may have increased practical significance [30]. Nonetheless, study findings should be confirmed in a larger cohort of neonates. Second, we were unable to compare changes in DNAm to an untreated comparison sample and cannot definitively conclude that changes in DNAm are solely due to pharmacologic treatment. It is possible that changes in NNNS and potentially in DNAm may be due to maturation, rather than pharmacologic treatment. Since neonates had DNAm and NNNS measured before and after treatment and because neonates treated longer were more likely to receive greater doses of morphine, both LOT and morphine dose are confounded by chronological age. Teasing apart the impact of treatment versus time would require selecting a comparison group(s) such as opioid exposed and untreated or unexposed neonates. Neonates exposed to opioids in utero who are 
treated for NAS likely differ in many ways from unexposed neonates and from those exposed to opioids who do not develop NAS. These difficulties may therefore not be easily addressed in future studies.

In conclusion, pharmacologic treatment for NAS is associated with decreased DNAm of OPRM1 and improved neonatal neurobehavior. These findings suggest that changes in DNAm may be one mechanism explaining how pharmacologic treatment for NAS improves neonatal neurobehavioral outcomes. 


\section{References}

[1] Haight SC, Ko JY, Tong VT, Bohm MK, Callaghan WM. Opioid Use Disorder Documented at Delivery Hospitalization — United States, 1999-2014. MMWR Morb Mortal Wkly Rep 2018;67:845-9. https://doi.org/10.15585/mmwr.mm6731a1.

[2] Patrick SW, Barfield WD, Poindexter BB. Neonatal Opioid Withdrawal Syndrome. Pediatrics 2020;146:e2020029074.

[3] Patrick SW, Schumacher RE, Benneyworth BD, Krans EE, McAllister JM, Davis MM. Neonatal Abstinence Syndrome and Associated Health Care Expenditures. JAMA 2012;307:1934-40. https://doi.org/10.1001/jama.2012.3951.

[4] Patrick SW, Davis MM, Lehmann CU, Cooper WO. Increasing incidence and geographic distribution of neonatal abstinence syndrome: United States 2009 to 2012. J Perinatol 2015;35:650-5. https://doi.org/10.1038/jp.2015.36.

[5] Winkelman TNA, Villapiano N, Kozhimannil KB, Davis MM, Patrick SW. Incidence and Costs of Neonatal Abstinence Syndrome Among Infants With Medicaid: 2004 - 2014. Pediatrics 2018;141:e20173520. https://doi.org/10.1542/peds.2017-3520.

[6] Leech AA, Cooper WO, McNeer E, Scott TA, Patrick SW. Neonatal Abstinence Syndrome In The United States, 2004-16. Health Aff 2020;39:764-7. https://doi.org/10.1377/hlthaff.2019.00814.

[7] Young LW, Hu Z, Annett RD, Das A, Fuller JF, Higgins RD, et al. Site-level variation in the characteristics and care of infants with neonatal opioid withdrawal. Pediatrics 2021;147. https://doi.org/10.1542/PEDS.2020-008839.

[8] Coyle MG, Ferguson A, Lagasse L, Liu J, Lester B. Neurobehavioral effects of treatment for opiate withdrawal. Arch Dis Child - Fetal Neonatal Ed 2005;90:F73-4. 
https://doi.org/10.1136/adc.2003.046276.

[9] Coyle MG, Salisbury AL, Lester BM, Jones HE, Lin H, Graf-Rohrmeister K, et al. Neonatal neurobehavior effects following buprenorphine versus methadone exposure. Addiction 2012;107:63-73. https://doi.org/10.1111/j.1360-0443.2012.04040.x.

[10] Heller NA, Logan BA, Morrison DG, Paul JA, Brown MS, Hayes MJ. Neonatal abstinence syndrome: Neurobehavior at 6 weeks of age in infants with or without pharmacological treatment for withdrawal. Dev Psychobiol 2017;59:574-82. https://doi.org/10.1002/dev.21532.

[11] Velez ML, McConnell K, Spencer N, Montoya L, Tuten M, Jansson LM. Prenatal buprenorphine exposure and neonatal neurobehavioral functioning. Early Hum Dev 2018;117:7-14. https://doi.org/10.1016/j.earlhumdev.2017.11.009.

[12] Wachman EM, Farrer LA. The genetics and epigenetics of Neonatal Abstinence Syndrome. Semin Fetal Neonatal Med 2019;24:105-10. https://doi.org/10.1016/j.siny.2019.01.002.

[13] Wachman EM, Hayes MJ, Lester BM, Terrin N, Brown MS, Nielsen DA, et al. Epigenetic Variation in the Mu-Opioid Receptor Gene in Infants with Neonatal Abstinence Syndrome. J Pediatr 2014;165:472-8. https://doi.org/10.1016/j.jpeds.2014.05.040.

[14] Wachman EM, Hayes MJ, Shrestha H, Nikita FNU, Nolin A, Hoyo L, et al. Epigenetic variation in OPRM1 gene in opioid-exposed mother-infant dyads. Genes, Brain Behav 2018;17:e12476. https://doi.org/10.1111/gbb.12476.

[15] Lester BM, Tronick EZ. History and Description of the Neonatal Intensive Care Unit Network Neurobehavioral Scale. Pediatrics 2004;113:634-40.

[16] Velez ML, Jansson LM, Schroeder J, Williams E. Prenatal Methadone Exposure and 
Neonatal Neurobehavioral Functioning. Pediatr Res 2009;66:704-9. https://doi.org/10.1203/PDR.0b013e3181bc035d.

[17] Jones HE, O'Grady KE, Johnson RE, Velez M, Jansson LM. Infant Neurobehavior Following Prenatal Exposure to Methadone or Buprenorphine: Results From the Neonatal Intensive Care Unit Network Neurobehavioral Scale. Subst Use Misuse 2010;45:2244-57. https://doi.org/10.3109/10826084.2010.484474.

[18] Lester BM, Tronick EZ, LaGasse L, Seifer R, Bauer CR, Shankaran S, et al. The Maternal Lifestyle Study: Effects of Substance Exposure During Pregnancy on Neurodevelopmental Outcome in 1-Month-Old Infants. Pediatrics 2002;110:1182-92. https://doi.org/10.1542/peds.110.6.1182.

[19] Jones HE, Kaltenbach K, Heil SH, Stine SM, Coyle MG, Arria AM, et al. Neonatal Abstinence Syndrome after Methadone or Buprenorphine Exposure. N Engl J Med 2010;363:2320-31. https://doi.org/10.1056/NEJMoa1005359.

[20] Padbury JF, Do BT, Bann CM, Marsit C, Hintz SR, Vohr BR, et al. DNA methylation in former extremely low birth weight newborns: association with cardiovascular and endocrine function. Pediatr Res 2021. https://doi.org/10.1038/s41390-021-01531-5.

[21] McLaughlin P, Mactier H, Gillis C, Hickish T, Parker A, Liang W-J, et al. Increased DNA Methylation of ABCB1 , CYP2D6, and OPRM1 Genes in Newborn Infants of Methadone-Maintained Opioid-Dependent Mothers. J Pediatr 2017;190:180-184.e1. https://doi.org/10.1016/j.jpeds.2017.07.026.

[22] Douet V, Heller MB, Le Saux O. DNA methylation and Sp1 binding determine the tissuespecific transcriptional activity of the mouse Abcc6 promoter. Biochem Biophys Res Commun 2007;354:66-71. https://doi.org/10.1016/j.bbrc.2006.12.151. 
[23] Michelotti GA, Brinkley DM, Morris DP, Smith MP, Louie RJ, Schwinn DA. Epigenetic regulation of human alpha1d-adrenergic receptor gene expression: a role for DNA methylation in Sp1-dependent regulation. FASEB J 2007;21:1979-93. https://doi.org/10.1096/fj.06-7118com.

[24] Oertel BG, Doehring A, Roskam B, Kettner M, Hackmann N, Ferreirós N, et al. Geneticepigenetic interaction modulates $\mu$-opioid receptor regulation. Hum Mol Genet 2012;21:4751-60. https://doi.org/10.1093/hmg/dds314.

[25] Flannery T, Davis JM, Czynski AJ, Dansereau LM, Oliveira EL, Camardo SA, et al. Neonatal Abstinence Syndrome Severity Index Predicts 18 Month Neurodevelopmental Outcome in Neonates Randomized to Morphine or Methadone. J Pediatr 2020;227:101107.e1. https://doi.org/10.1016/j.jpeds.2020.08.034.

[26] Leyenaar JK, Schaefer AP, Wasserman JR, Moen EL, O’Malley AJ, Goodman DC. Infant Mortality Associated with Prenatal Opioid Exposure. JAMA Pediatr 2021;03756:1-9. https://doi.org/10.1001/jamapediatrics.2020.6364.

[27] Liu J, Bann C, Lester B, Tronick E, Das A, Lagasse L, et al. Neonatal Neurobehavior Predicts Medical and Behavioral Outcome. Pediatrics 2010;125:e90-8. https://doi.org/10.1542/peds.2009-0204.

[28] Everson TM, Marsit CJ, Michael O’Shea T, Burt A, Hermetz K, Carter BS, et al. Epigenome-wide Analysis Identifies Genes and Pathways Linked to Neurobehavioral Variation in Preterm Infants. Sci Rep 2019;9:1-13. https://doi.org/10.1038/s41598-01942654-4.

[29] Lester BM, Marsit CJ, Giarraputo J, Hawes K, LaGasse LL, Padbury JF. Neurobehavior related to epigenetic differences in preterm infants. Epigenomics 2015;7:1123-36. 
https://doi.org/10.2217/epi.15.63.

[30] Cohen J. Statistical Power Analysis for the Behavioral Sciences. Hillsdale: Erlbaum; 1988. 
Table 1

Maternal and Neonatal Characteristics

\begin{tabular}{|c|c|c|}
\hline Variable & $\mathbf{N}$ & M $(95 \% \mathrm{CI})$ or $\mathrm{N}(\%)$ \\
\hline Maternal age (years) & 37 & $29.4(27.8-31.0)$ \\
\hline \multicolumn{3}{|l|}{ Med Assisted Treatment } \\
\hline Methadone & 37 & $16(43 \%)$ \\
\hline Buprenorphine & 37 & $21(57 \%)$ \\
\hline \multicolumn{3}{|l|}{ Concurrent Exposures } \\
\hline Cigarette Smoking & 36 & $27(75 \%)$ \\
\hline Alcohol & 37 & $1(3 \%)$ \\
\hline Benzodiazepines & 35 & $4(11 \%)$ \\
\hline SSRIs & 35 & $6(7 \%)$ \\
\hline Male sex & 37 & $20(54 \%)$ \\
\hline Caucasian & 36 & $31(86 \%)$ \\
\hline Gestational age (weeks) & 37 & $38.9(38.5-39.2)$ \\
\hline Birth weight (grams) & 37 & $3,011(2,884-3,138)$ \\
\hline Head circumference $(\mathrm{cm})$ & 37 & $34.0(33.6-34.4)$ \\
\hline \multicolumn{3}{|l|}{ Feeding } \\
\hline Breastfed (any) & 37 & $21(57 \%)$ \\
\hline Formula only & 37 & $16(43 \%)$ \\
\hline
\end{tabular}


Table 2

NAS treatment

\begin{tabular}{lcc}
\hline Variable & N & M (95\% CI) or N (\%) \\
\hline Length of treatment (days) & 37 & $14.2(12.7-15.8)$ \\
Length of stay due to NAS (days) & 37 & $17.2(15.7-18.8)$ \\
Maximum Finnegan score & 37 & $11.6(11.0-12.2)$ \\
Morphine, total dose (mg) & 37 & $13.2(10.5-15.9)$ \\
Phenobarbital use & 37 & $21(57 \%)$ \\
\hline
\end{tabular}


Table 3

DNA methylation and neurobehavior before and after pharmacological treatment

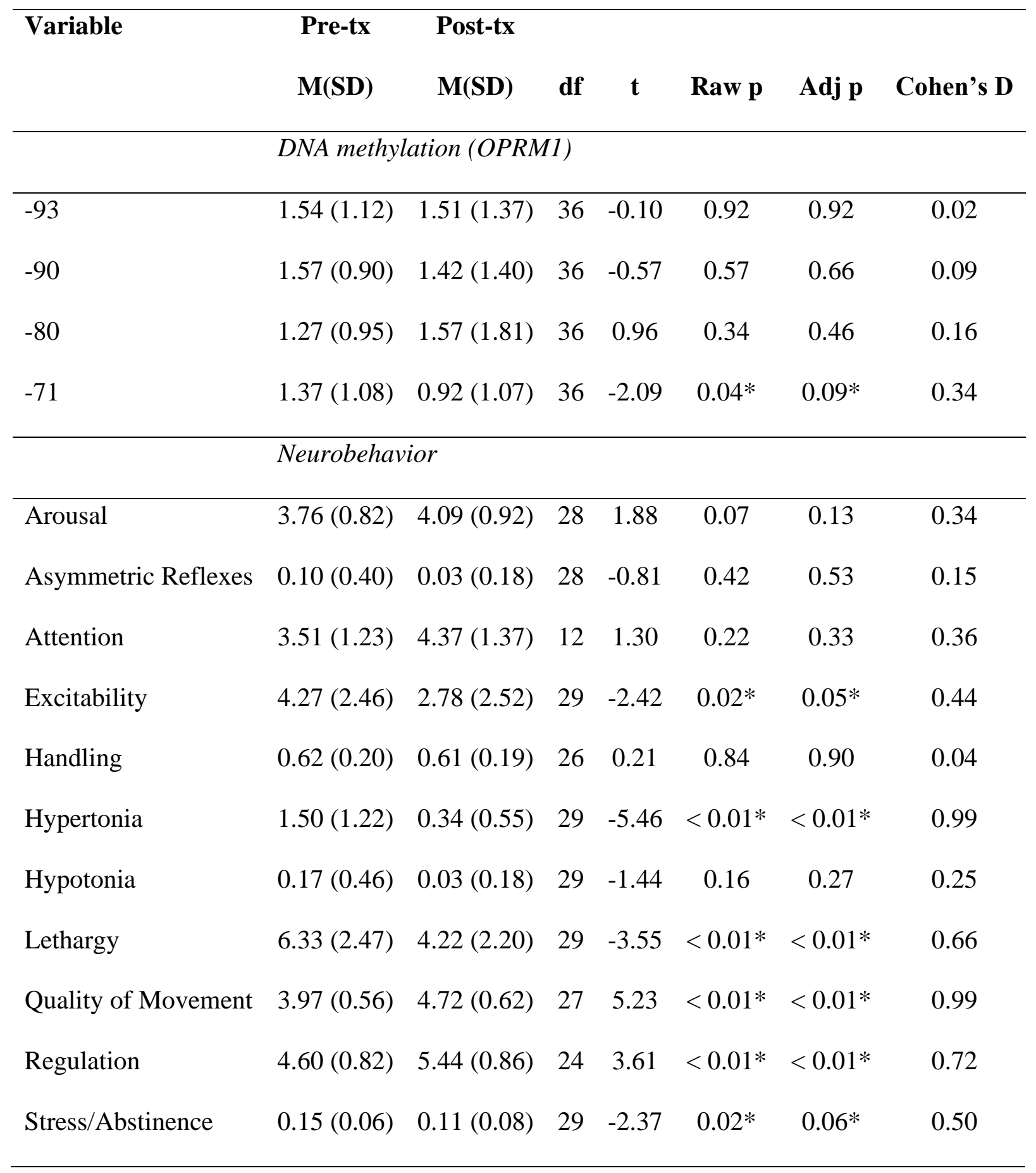

Note. $*$ denotes p-values that are significant with a $10 \%$ false discovery rate (FDR). 
Table 4

\section{Summary of Path Coefficients}

\begin{tabular}{|c|c|c|c|c|}
\hline Path & $\beta(\mathrm{SE})$ & $\beta(\mathrm{SE})$ & $\beta(\mathbf{S E})$ & $\beta(\mathbf{S E})$ \\
\hline Length of Treatment (LOT) & LOT $\rightarrow$ DNAm & DNAm $\rightarrow$ NNNS & LOT $\rightarrow$ NNNS & LOT $\rightarrow$ DNAm $\rightarrow$ NNNS \\
\hline Excitability & $-.29 *$ & $.24+$ & $-.34 *$ & -.07 \\
\hline Hypertonia & $-.29 *$ & $.37 * * *$ & .09 & $-.11+$ \\
\hline Lethargy & $-.29 *$ & $-.31 *$ & -.19 & .09 \\
\hline Quality of Movement & $-.29 *$ & -.20 & .10 & .06 \\
\hline Regulation & $-.29 *$ & -.26 & .24 & .07 \\
\hline Stress/Abstinence & $-.29 *$ & .08 & $-.43^{*}$ & -.02 \\
\hline Morphine, total dose (MOR) & MOR $\rightarrow$ DNAm & DNAm $\rightarrow$ NNNS & $\mathrm{MOR} \rightarrow \mathrm{NNNS}$ & $\mathrm{MOR} \rightarrow \mathrm{DNAm} \rightarrow \mathrm{NNNS}$ \\
\hline Excitability & $-.31 *$ & $.40 * *$ & .06 & $-.13 *$ \\
\hline Hypertonia & $-.31 *$ & $.45 * * *$ & .27 & $-.14+$ \\
\hline Lethargy & $-.31 *$ & $-.37 *$ & $-.36 * *$ & $.12+$ \\
\hline Quality of Movement & $-.31 *$ & $-.32+$ & -.19 & .10 \\
\hline Regulation & $-.31 *$ & -.35 & .03 & .11 \\
\hline Stress/Abstinence & $-.31 *$ & .22 & -.10 & -.07 \\
\hline
\end{tabular}

Note. $* p<.05, * * p<.01, * * * p<.001$ 